\title{
Human Basal Ganglia and the Dynamic Control of Force during On-Line Corrections
}

\author{
Scott T. Grafton ${ }^{1}$ and Eugene Tunik ${ }^{2}$ \\ ${ }^{1}$ Sage Center for the Study of Mind, Department of Psychology, University of California, Santa Barbara, California 93106, and ${ }^{2}$ Department of Rehabilitation \\ and Movement Science, University of Medicine and Dentistry of New Jersey, Newark, New Jersey 07107
}

\begin{abstract}
Natural movements are corrected in part by the generation of submovements, occurring early in a movement such that they amend an ongoing action. Submovements are associated with activity of the basal ganglia, implying a role for the structures in error correction. In parallel, the basal ganglia are linked to the generation and control of force amplitude, change, and duration. Here, we tested whether activity in human basal ganglia is associated with submovements generally, or was specific to a condition where the submovements only occurred in the face of unexpected proprioceptive error. Submovements were induced by introducing unexpected and variable viscous loads (augmenting the need for trial-specific grip forces) or by reducing target size (augmenting the need for visually guided on-line control) in a one-dimensional target-capture task. In both cases, subjects compensated for the increased task difficulty by generating corrective submovements, which were closely matched in frequency and type. Activity in the internal segment of the globus pallidus and subthalamic nucleus correlated strongly with the number of submovements during the viscous challenge but not with the target challenge. The effects could not be explained by kinematic differences, i.e., movement amplitude or average number of submovements. The results support a specific role for the basal ganglia in error correction under conditions of variable load where there is a need for the dynamic control of force within an ongoing movement.
\end{abstract}

\section{Introduction}

Even under the best of conditions, the control of visually guided behavior requires on-line corrections to reconcile performance errors. On-line corrections can be observed early in a movement, suggesting they rely on an internal model of expected plant dynamics (Kawato, 1999; Desmurget and Grafton, 2000). One approach for detecting early error correction is the identification of submovements in the kinematic trace during onedimensional (1-D) target capture. Submovements are identified as distinct peaks in the velocity trace (Novak et al., 2000, 2002), modeled as small, bell-shaped velocity profiles either superimposed onto or occurring after the primary movement (Fishbach et al., 2005). Single-unit physiological recordings of monkeys performing 1-D target capture demonstrate a modulation of neuronal activity in the internal segment of the globus pallidus (GPi) in association with submovement generation (Roy et al., 2008). In a 1-D target capture task in the presence of unpredictable viscous loads, human imaging shows a correlation of putamen activity with the number of submovements per trial (Tunik et al., 2009).

In parallel, there is strong functional imaging evidence that the human basal ganglia (BG) play a role in controlling many kinematic properties, including movement velocity and ampli-

\footnotetext{
Received June 25, 2010; revised Nov. 17, 2010; accepted Dec. 1, 2010.

This work was supported by Public Health Service Grants NS44393 and K01 HD059983. We thank Jim Houk for helpful discussions.

Correspondence should be addressed to Dr. Scott T. Grafton, Department of Psychology, University of California Santa Barbara, Santa Barbara, CA 93106. E-mail: grafton@psych.ucsb.edu.

DOI:10.1523/JNEUROSCI.3301-10.2011

Copyright $\odot 2011$ the authors $\quad 0270-6474 / 11 / 311600-06 \$ 15.00 / 0$
}

tude in putamen and GPi (Turner et al., 1998, 2003; Spraker et al., 2007), force duration in putamen (Vaillancourt et al., 2004; Prodoehl et al., 2008) and force rate of change and amplitude in GPi and subthalamic nucleus (STN) (Spraker et al., 2007; Prodoehl et al., 2008). Of note, behavioral experiments show that both agonist and antagonist muscles will generate increased grip force in response to mechanical perturbations (Koshland and Hasan, 2000; Spraker et al., 2007), including those used in 1-D target capture.

Given these two lines of evidence, it is unclear whether the correlation of submovements with BG activity observed in humans (Tunik et al., 2009) is a general property of error correction or a specific product of generating forces to overcome viscous perturbations. The goal of the current study was to reconcile these alternative hypotheses. We focused specifically on parametric differences of activity in globus pallidus and putamen. To test the alternatives, half the trials were performed with a viscous challenge, where a torque motor applied resistive forces that varied unpredictably in severity from trial to trial. The other trials used a target challenge, where the motor was off and hence reactive grip forces were relatively constant across trials and submovements. Instead, the task was made difficult by varying the target width unpredictably across trials (Fitts, 1954). The two tasks were adaptively matched for movement amplitude and the number of submovements across trials. The critical prediction was that the BG would only scale with the number of submovements under the viscous challenge task, demonstrating specificity of the BG for generating force pulses during error correction. As an exploratory analysis, the specific and general hypotheses were also tested in the cerebellum and cortex. 
Viscous Challenge

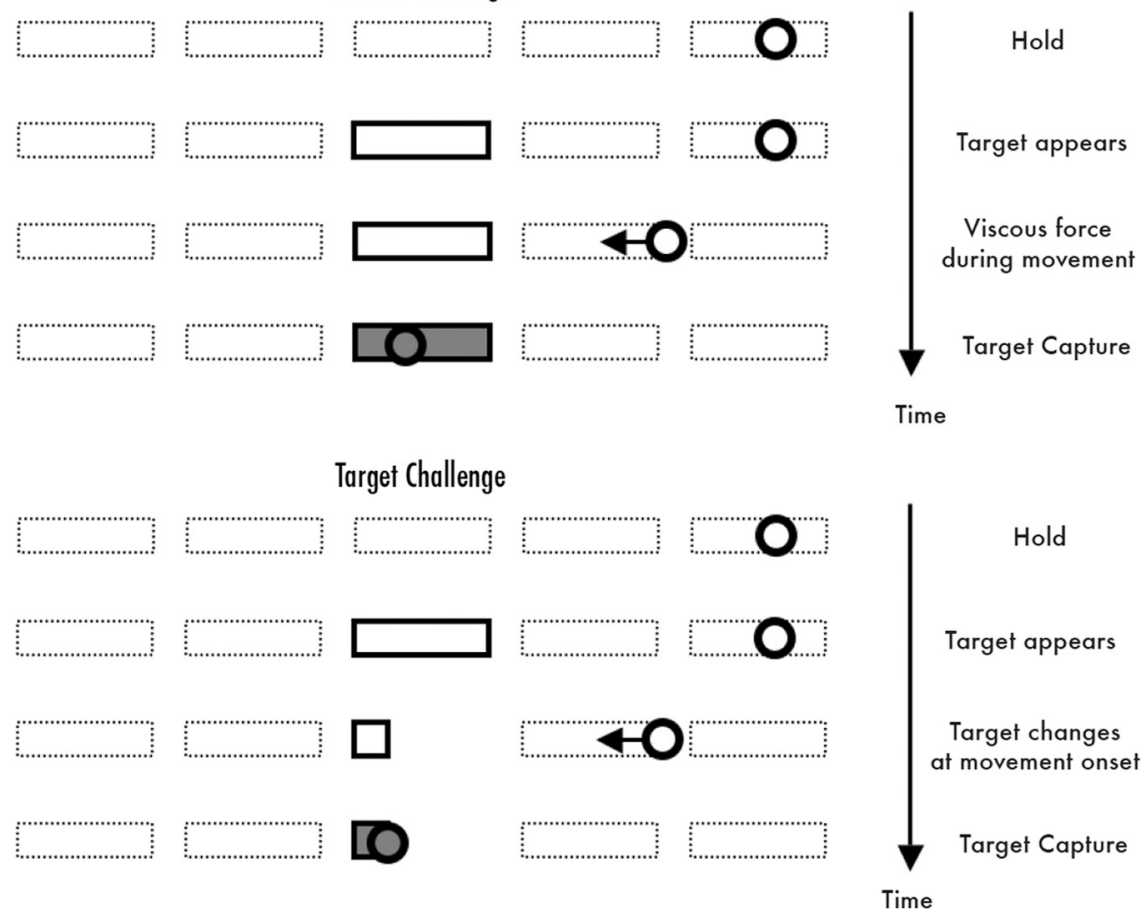

Figure 1. A trial started with the cursor (circle) held stationary in a target (dotted box). Then a new target (solid box) boundary was illuminated and subjects had to shift the cursor into this new position as quickly as possible. Once the cursor was positioned over a target, the latter became color filled. Movement direction and amplitude were randomly distributed across trials. In viscous challenge trials, a different viscous force was applied on each trial, and the target size was constant. In target challenge trials, there was no viscous force but the size of the target varied from trial to trial.

\section{Materials and Methods}

Subjects. Twenty-four right-handed (Oldfield, 1971) healthy young adults (mean age, 23 years; range, 18-33 years; 12 male, 12 female) volunteered for the study after informed consent was obtained in accordance with the University of California Santa Barabara Human Subjects Committee.

MRI. Functional MRI (fMRI) recordings were conducted using a 3T TIM Trio Siemens Magnetom with a 12-channel phased-array head coil. Foam padding was used for head stabilization. Each subject completed six functional runs using an echo planar gradient-echo imaging sequence sensitive to blood oxygenation level-dependent (BOLD) contrast with 33 slices per repetition time (TR) ( $3 \mathrm{~mm}$ thickness, $0.5 \mathrm{~mm}$ gap), $236 \mathrm{TRs}$, and a TR of $2000 \mathrm{~ms}$, echo time of $30 \mathrm{~ms}$, flip angle of $90^{\circ}$, field of view of $192 \mathrm{~mm}$, $64 \times 64$ matrix (voxel resolution, $3 \times 3 \times 3.5 \mathrm{~mm}$ ). A gradient field map of the same image size was obtained to correct for geometric distortions. A high-resolution T1-weighted sagittal MPRAGE sequence of the whole brain was acquired for anatomic localization.

Task. Participants performed the target-capture task using their right hand. They held a long PVC rod (with a comfortable wooden dowel handle) attached to a torque motor and position-sensing hardware. The torque motor device and radiofrequency shielding have been described previously (Tunik et al., 2007b, 2009). Participants viewed a backprojected flat screen $102 \mathrm{~cm}$ from the eyes, subtending $50^{\circ}$ horizontal view with five rectangular $\left(1^{\circ} \times 6^{\circ}\right)$ targets aligned horizontally every $10^{\circ}$. Horizontal position of a $1^{\circ}$ circular cursor was controlled by 1 -D forearm rotation and used to capture targets appearing in random order. The timing of events is shown in Figure 1. A trial started with the cursor held stationary in a target. Then a new target boundary was illuminated and subjects had to shift the cursor into this new position. Subjects were told to go as quickly as possible. The cursor became invisible at movement onset. Whenever the cursor was positioned over the target, the latter instantly became color-filled and would decolor if the cursor overshot the target. The cursor reappeared once it was stationary over a target for $300 \mathrm{~ms}$ or when a trial timed out $(1500 \mathrm{~ms})$. The position of the reap- pearing cursor provided knowledge of results and became the starting position for the subsequent trial. The average intertrial interval was $8.1 \mathrm{~s}$ and varied from 5.2 to $12.8 \mathrm{~s}$. Fifty trials were acquired per scan, with a total of 300 trials. In three scans of 50 trials each, a torque motor was programmed to provide a positive, velocity-dependent (viscous) torque field proportional to the subjects' velocity. Torque strength was randomly varied on a trial-by-trial basis with a maximum of $-0.5 \mathrm{oz}-\mathrm{in} /{ }^{\circ} \mathrm{s}{ }^{-1}(0.004$ $\mathrm{Nm} /{ }^{\circ} \mathrm{s}^{-1}$ ) (Tunik et al., 2007b, 2009). In the three other scans of 50 trials each, the torque field was off, but the width of the target was randomly varied between $3^{\circ}$ and $9^{\circ}$ from trial to trial. In pilot testing, an adaptive algorithm was used to define a range of target widths so that the viscous and target challenge tasks were balanced in the distribution and number of submovements. The ordering of viscous and target challenge scans was randomized across subjects.

Performance analysis. Cursor position was sampled at $1500 \mathrm{~Hz}$ and smoothed offline with a Butterworth low-pass filter at $10 \mathrm{~Hz}$. The position data were differentiated to yield a velocity trace. Movement onset was defined as the time at which the angular velocity exceeded and remained $>5 \%$ of the peak angular velocity for $>100 \mathrm{~ms}$. Movement offset was defined as the time at which the knob angle did not change by $>5^{\circ}$ for $>0.4$ s. Movement time was defined as the interval between movement onset and offset. Movement amplitude was defined as the displacement (in angular terms) between the angle at the start and the maximal angular displacement in the corresponding trial. Each movement was decomposed into a primary movement and any submovements by computing the third derivative of the position (jerk) and identifying all zero crossings occurring between the movement onset and offset. The number of zero crossings was divided by two to determine the total number of movements (velocity peaks) in a given trajectory. Subtracting the single primary movement from this number determined the number of submovements. The proportion of submovements overlapping with the primary movement was estimated as described previously (Tunik et al., 2009). Peak velocity and peak acceleration of the primary movement and time to first submovement were derived from the velocity and acceleration trace. Statistical tests were determined by paired $t$ test after averaging all trials within each condition per subject. Significance was adjusted for six multiple pairwise two-way comparisons of the kinematic measures (threshold, $p<0.008$ ).

Image analysis. BOLD fMRI was preprocessed in SPM 5 by correcting functional scans for geometric distortion (Jezzard and Balaban, 1995) using the FieldMap V2.0 toolbox available in SPM 5, registering fMRI data within runs to each other, fitting this realigned fMRI data to each subjects high-resolution scan, spatially normalizing the anatomic and fMRI data to the canonical T1 template in SPM 5, and spatially smoothing the resultant functional data with an $8 \mathrm{~mm}$ Gaussian filter. The cerebellum and brainstem were spatially normalized separately (Diedrichsen, 2006).

Event estimation. All trials were included in the analyses. Conditionspecific components of the BOLD signal were identified with a general linear model and event-related fMRI. A design matrix was created for each subject with vectors containing the onset and duration of each movement, coded separately for each viscous or target challenge run. Vectors for the number of submovements per trial and movement amplitude (each adjusted to create a zero mean vector) were included separately for each run. This made it possible to correlate the mean number of submovements with the corresponding magnitude of the BOLD response on a trial-by-trial basis independent of movement related activity. Temporal derivatives for task, submovement, and amplitude events were 

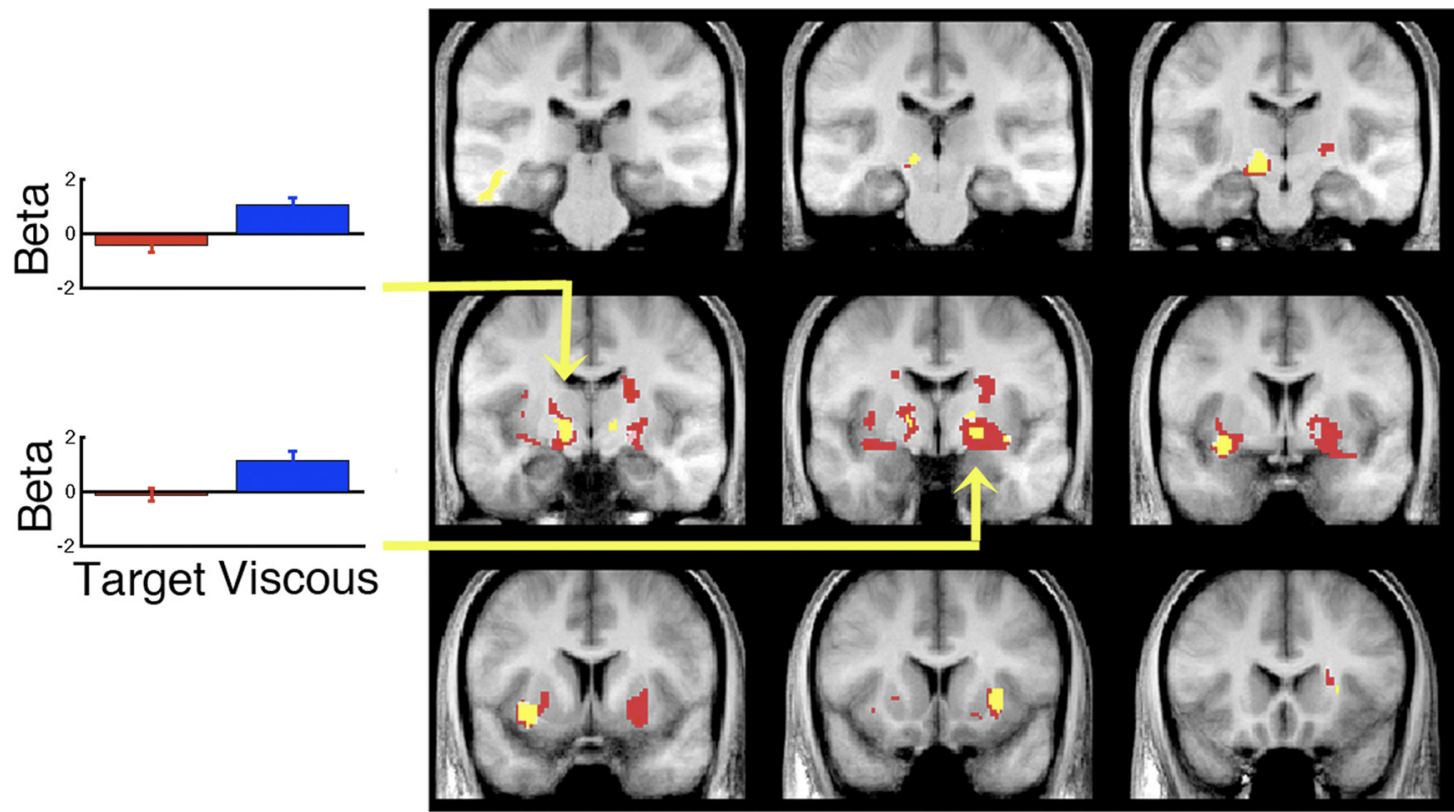

Figure 2. Correlation of trial-by-trial submovements and BOLD activity in basal ganglia. Significant positive correlations for submovements at the group level, corrected for multiple test comparisons ( FDR, $p<0.05)$ are shown in red for the viscous trials and blue for the target trials. Significant differences where the correlation with submovements was stronger for viscous than target challenge task are shown in yellow. Left, Mean beta \pm SEM for the entire population corresponding to the magnitude of the parameter correlating submovement and BOLD for the left GPi/STN (MNI: $-14,-18,-6)$ and right GPi (MNI: $20,-10,-8)$. Coronal sections are $-30,-23,-16,-11,-4,2,9,13$, and $20 \mathrm{~mm}$ relative to anterior commissure.

included, along with blocking factors of noninterest to denote different scan runs. The design matrix was convolved with a canonical hemodynamic response function defined in SPM 5 and beta weights were estimated with the general linear model using restricted weighted least squares (Diedrichsen and Shadmehr, 2005).

Contrast estimation. For each subject, the following contrasts were generated. First, an all trial (viscous + target) versus baseline contrast was generated to identify all task related areas. This contrast [with a threshold controlling for a false discovery rate (FDR) of $p<0.05$ to account for multiple testing] was used as an inclusive mask for all subsequent analyses of effects in the cortex (supplemental Fig. 1, available at www.jneurosci.org as supplemental material). Second, correlations between the number of submovements and BOLD activity were calculated separately for viscous and target challenge tasks. Third, task-specific differences between the correlation of BOLD activity with submovements were calculated by contrasting the viscous and target task parametric correlations with each other. Fourth, correlations between amplitude and BOLD activity were calculated separately for viscous and target challenge tasks. Fifth, task-specific differences between the correlations of BOLD activity with amplitude were calculated by contrasting the two task-specific correlations.

Population estimation. The above contrast images were entered into random-effects analyses to characterize population-based effects. Based on the a priori anatomic hypothesis, a BG volume was drawn as a single, contiguous, anatomically defined, large region of interest covering the striatum, insula, internal capsule, pallidum, and midbrain. Task differences of submovement and amplitude modulation of BOLD activity in this region was tested at a threshold of $p<0.05$, FDR, corrected for search volume. A second, anatomically defined, large region of interest covering the upper half of the cerebellum and brainstem was analyzed both with
FDR correction and at $p<0.01$, uncorrected, for exploratory purposes. Task differences in the cortex were tested at a threshold of $p<0.05$, FDR-corrected, within a mask defined by all trials versus rest.

\section{Results}

\section{Behavior}

Differences of movement kinematics were compared for trials performed under a viscous and target challenge. After correcting for multiple comparisons, there were no significant differences in the movement amplitude (viscous, $23.0^{\circ} \pm 0.2^{\circ}$; target, $22.7^{\circ} \pm$ $0.2^{\circ}$ ), time to peak acceleration (viscous, $218.9 \pm 19.0^{\circ} / \mathrm{s}^{2}$; target, 


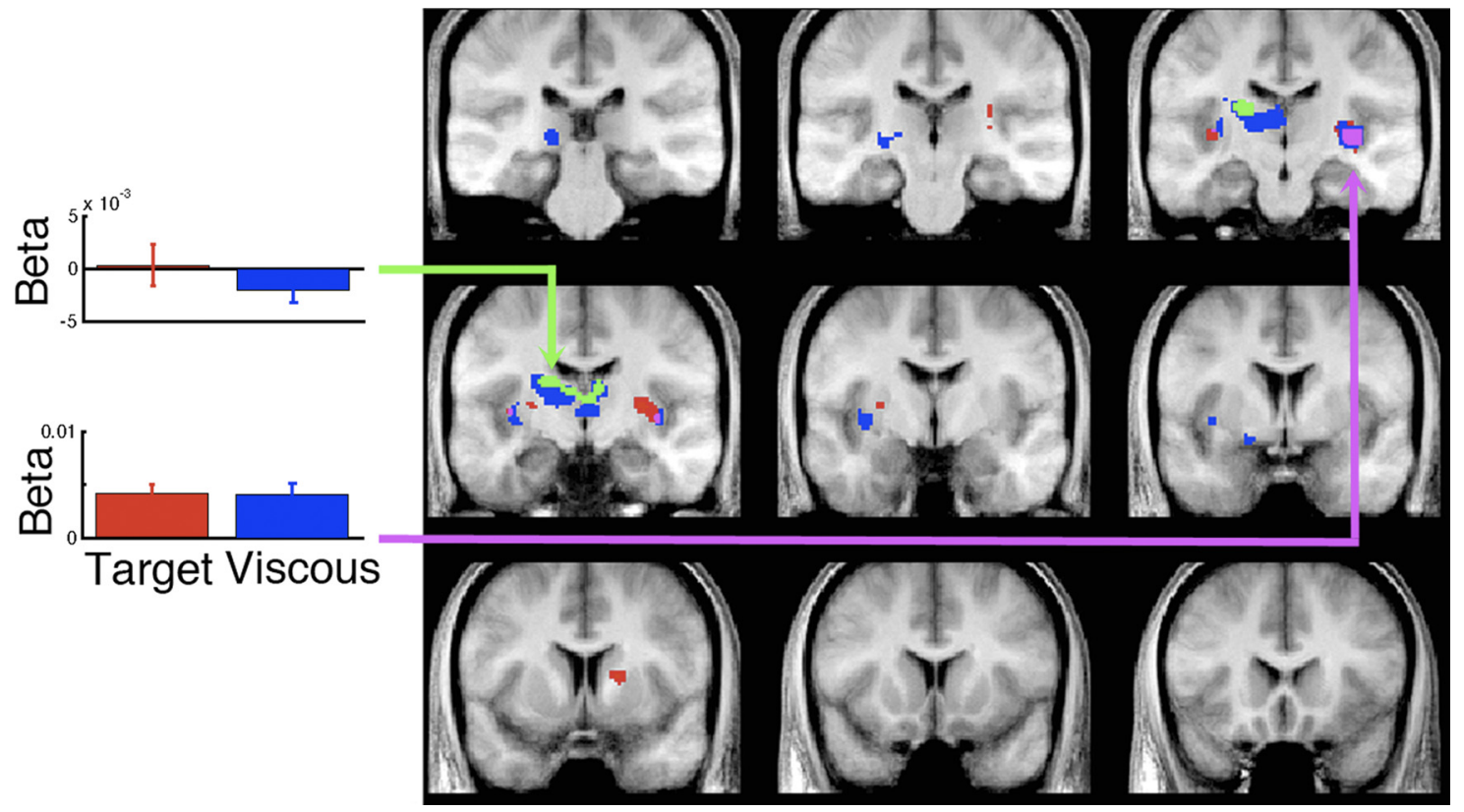

Figure 4. Correlation of trial-by-trial movement amplitude and BOLD activity in basal ganglia. Significant positive correlations with amplitude at the group level, corrected for multiple test comparisons (FDR, $p<0.05$ ) are shown in red for the viscous trials, blue for the target trials, and purple for their overlap. Significant task differences where the correlation with amplitude was stronger for target than viscous challenge task are shown in green. Left, Mean beta \pm SEM for the entire population corresponding to the magnitude of the parameter correlating amplitude and BOLD for the left caudate (MNI: -14,-12,22) and right putamen/insula (MNI: 34, -20,0). Coronal sections are $-30,-23,-16,-11,-4,2,9,13$, and $20 \mathrm{~mm}$ relative to anterior commissure.

$257.6 \pm 23.9 \% \mathrm{~s}^{2}$ ), the number of submovements (viscous, $2.66 \pm$ 0.17 ; target, $2.45, \pm 0.23$ ), or percentage of overlapping (viscous, 91.6\%; target, 93.5\%), discrete (viscous, $7.6 \%$; target, $7.1 \%$ ), or reversing (viscous, $1.2 \%$; target, $1 \%$ ) submovements. The distributions in the movement amplitude and in the number of submovements were similar for the two types of trials (supplemental Fig. 2, available at www.jneurosci.org as supplemental material). The time to onset for the first overlapping submovement was significantly faster for viscous $(149.0 \pm 5.2 \mathrm{~ms})$ than target $(175.2 \pm 6.8 \mathrm{~ms})$ trials $(t$ test, $6.51 ; p<0.001$; df, 23). These compensatory response latencies are consistent with those reported in other on-line perturbation paradigms (Day and Marsden, 1982; Prablanc and Martin, 1992). Peak velocity (viscous, $35.0 \pm 2.5^{\circ} / \mathrm{s}$; target, $45.7 \pm 3.6^{\circ} / \mathrm{s} ; t$ test, $4.23 ; p<0.004 ; \mathrm{df}, 23$ ) and total time to complete a movement (viscous, $1.39 \pm 0.01 \mathrm{~s}$; target, $1.47 \pm 0.01 \mathrm{~s} ; t$ test, $11.07 ; p<0.001$; df, 23) were significantly less for the viscous than the target task. In summary, there was no difference in the frequency of submovements between tasks. There was a small difference in the timing of submovement generation, likely related to the proprioceptive feedback available in the viscous task. There was no evidence for greater velocity or acceleration in generating the primary movement in the viscous condition.

\section{Basal ganglia activity during on-line correction}

Image analysis was designed to identify where there was a positive correlation between the number of submovements generated per trial and BOLD activity in basal ganglia nuclei for the viscous, but not the target, challenge.

At the group level, a random-effects analysis corrected for multiple test comparisons demonstrated multiple sites in bilateral GPi, STN, and putamen that correlated with the number of submovements in the viscous challenge task (Fig. 2, red). Critically, there was no positive correlation between submovements and activity in the same BG regions when subjects performed the target challenge task. These different effects of viscous and target challenge on basal ganglia activity and submovements was confirmed by comparing these parametric correlations directly. There was a significantly stronger correlation in the left GPi and STN with submovements formed with a viscous than target challenge (Fig. 2, yellow). The exact loci and effect sizes for all of these areas are summarized in supplemental Table 1 (available at www. jneurosci.org as supplemental material). This pattern of activity is shown for activity in the left GPi/STN from a representative subject in Figure 3. In addition, note that in the same region, there is no correlation with overall movement amplitude for either task. Although movement duration differed between tasks, this was modeled explicitly for each trial in the image analysis and not likely to explain these effects.

The amplitude of a movement correlated significantly with activity in both caudate and putamen, with a left-sided predominance (Fig. 4). This was true for both the target and viscous challenge tasks. They overlapped in the right putamen/insula. There was a stronger correlation between amplitude and BOLD activity for the target challenge task in a small portion of left dorsal putamen and caudate nucleus. Of note, none of the correlations of amplitude and BOLD activity in the basal ganglia overlapped spatially with the BOLD correlates with submovements (Fig. 2).

Cerebellar and cortical activity during on-line correction Within the cerebellum, there was no significant correlation between the number of submovements and BOLD activity for either task. Nor was there a significant difference between tasks. Within the cortex, there was a significant correlation between the number of submovements and BOLD activity in the viscous challenge task located within the bilateral intraparietal sulcus and right inferior parietal lobule, as shown in supplemental Figure 3 (available at www.jneurosci.org as supplemental material).

Within the right anterior cerebellar cortex there was a modest correlation of activity with movement amplitude for both tasks with relatively greater amplitude modulation for the viscous than 
target challenge tasks. Within the cortex, both tasks showed a correlation between movement amplitude and activity in left premotor, motor, and superior parietal cortices; supplementary motor area, and cingulate motor area, as shown in supplemental Figure 4 (available at www.jneurosci.org as supplemental material).

\section{Discussion}

The main finding of this study was a selective correlation between the number of submovements generated per trial with activity in bilateral GPi and left STN, but only under conditions of a variable viscous load. This association was not observed when submovements were generated in the face of error due to difficult targeting requirements. This interaction of task and parametric modulation cannot be attributed to the main effect when comparing the two tasks directly, which by definition will differ in at least some measures of kinematics and task requirements. Indeed, although we observed modest kinematic differences in peak velocity and peak amplitude of the primary movement, these were greater for the target challenge task and this is unlikely to explain the critical association of submovement and viscous load. It is also unlikely that the small difference in the time to first submovement $(28 \mathrm{~ms})$ is sufficiently large to explain these parametric effects, given the insensitivity of fMRI due to the smooth hemodynamic response function for detecting differences at such a fine time resolution. In summary, the parametric correlation approach adopted here allows us to establish the specific role of BG in submovement formation in the face of proprioceptive loads.

The dissociation between the viscous and target challenge tasks on submovement formation suggests that the GPi and STN are involved in the on-line control of a movement under conditions where force control is particularly important. Whether this is related to detection/correction processes or force generation cannot be inferred directly as grip force was not measured. However, a number of human studies are consistent with the latter interpretation. Specifically, when challenged with a viscous perturbation, there is a well defined increase of force in agonist and antagonist muscles, regardless of movement amplitude or duration (Koshland and Hasan, 2000). Thus, in our task, each submovement that was generated in the face of a viscous load led to a transient increase in grip force. The involvement of GPi and STN in this process extends recent studies showing that under conditions of dynamic, continuous control, the rate of change of grip force, as well as grip force amplitude, correlates with activity in the same areas observed in the current experiment (Spraker et al., 2007; Prodoehl et al., 2009). The present results allow us to suggest a more refined role for the basal ganglia in the on-line control of error corrections. Although there is growing evidence from patient experiments that on-line corrections for motor perturbations are impoverished after dopamine depletion (Tunik et al., 2004, 2007a), perhaps due to the basal ganglia's reliance on an internal model to make corrections, the current results show that this process is intertwined with the precise generation of forces needed as part of the overall correction.

The lack of correlations between GPi/STN activity and submovements in the target challenge task shows that there are different forms of error correction or force production determined by unique task demands. We speculate that in the target challenge task, the main source of detected error is visual rather than proprioceptive or kinesthetic. This would also explain the slower time to first submovement in the target challenge. Even though our task used only 1-D movements, visual information could be used to define a difference vector error that is minimized on-line with motor output that does not require modulation of grip force that is tailored to each submovement, as in the viscous case. Previous studies of visuomotor control support a role for parietal premotor cortices and cerebellum in visually guided error correction during reaching, consistent with the current results (Desmurget et al., 1999, 2001; Diedrichsen et al., 2005).

The second main finding was a correlation between movement amplitude and activity in the putamen and anterior cerebellum. This was true for both the viscous and target challenge task. This finding extends previous studies of movement amplitude based on tomographic blood flow scans (Turner et al., 2003) as well as fMRI studies examining the interaction of movement duration and force (Vaillancourt et al., 2004; Prodoehl et al., 2009). Large amplitude movements require force to be generated for a longer duration. The current results show this is true for all forces, i.e., whether there is a viscous load present or not.

\section{References}

Day BL, Marsden CD (1982) Accurate repositioning of the human thumb against unpredictable dynamic loads is dependent upon peripheral feedback. J Physiol 327:393-407.

Desmurget M, Grafton S (2000) Forward modeling allows feedback control for fast reaching movements. Trends Cogn Sci 4:423-431.

Desmurget M, Epstein CM, Turner RS, Prablanc C, Alexander GE, Grafton ST (1999) Role of the posterior parietal cortex in updating reaching movements to a visual target. Nat Neurosci 2:563-567.

Desmurget M, Gréa H, Grethe JS, Prablanc C, Alexander GE, Grafton ST (2001) Functional anatomy of nonvisual feedback loops during reaching: a positron emission tomography study. J Neurosci 21:2919-2928.

Diedrichsen J (2006) A spatially unbiased atlas template of the human cerebellum. Neuroimage 33:127-138.

Diedrichsen J, Shadmehr R (2005) Detecting and adjusting for artifacts in fMRI time series data. Neuroimage 27:624-634.

Diedrichsen J, Hashambhoy Y, Rane T, Shadmehr R (2005) Neural correlates of reach errors. J Neurosci 25:9919-9931.

Fishbach A, Roy SA, Bastianen C, Miller LE, Houk JC (2005) Kinematic properties of on-line error corrections in the monkey. Exp Brain Res 164:442-457.

Fitts PM (1954) The information capacity of the human motor system in controlling the amplitude of movement. J Exp Psychol 47:381-391.

Jezzard P, Balaban RS (1995) Correction for geometric distortion in echo planar images from B0 field variations. Magn Reson Med 34:65-73.

Kawato M (1999) Internal models for motor control and trajectory planning. Curr Opin Neurobiol 9:718-727.

Koshland GF, Hasan Z (2000) Electromyographic responses to a mechanical perturbation applied during impending arm movements in different directions: one-joint and two-joint conditions. Exp Brain Res 132:485-499.

Novak KE, Miller LE, Houk JC (2000) Kinematic properties of rapid hand movements in a knob turning task. Exp Brain Res 132:419-433.

Novak KE, Miller LE, Houk JC (2002) The use of overlapping submovements in the control of rapid hand movements. Exp Brain Res 144:351-364.

Oldfield RC (1971) The assessment and analysis of handedness: the Edinburgh inventory. Neuropsychologia 9:97-113.

Prablanc C, Martin O (1992) Automatic control during hand reaching at undetected two-dimensional target displacements. J Neurophysiol 67:455-469.

Prodoehl J, Yu H, Wasson P, Corcos DM, Vaillancourt DE (2008) Effects of visual and auditory feedback on sensorimotor circuits in the basal ganglia. J Neurophysiol 99:3042-3051.

Prodoehl J, Corcos DM, Vaillancourt DE (2009) Basal ganglia mechanisms underlying precision grip force control. Neurosci Biobehav Rev 33:900-908.

Roy SA, Tunik E, Bastianen C, Fishbach A, Grafton ST, Houk JC (2008) Firing patterns of GPi neurons associated with primary movements and corrective submovements. Soc Neurosci Abstr 34:378.9.

Spraker MB, Yu H, Corcos DM, Vaillancourt DE (2007) Role of individual 
basal ganglia nuclei in force amplitude generation. J Neurophysiol 98:821-834.

Tunik E, Adamovich SV, Poizner H, Feldman AG (2004) Deficits in rapid adjustments of movements according to task constraints in Parkinson's disease. Mov Disord 19:897-906.

Tunik E, Feldman AG, Poizner H (2007a) Dopamine replacement therapy does not restore the ability of Parkinsonian patients to make rapid adjustments in motor strategies according to changing sensorimotor contexts. Parkinsonism Relat Disord 13:425-433.

Tunik E, Schmitt PJ, Grafton ST (2007b) BOLD coherence reveals segregated functional neural interactions when adapting to distinct torque perturbations. J Neurophysiol 97:2107-2120.
Tunik E, Houk JC, Grafton ST (2009) Basal ganglia contribution to the initiation of corrective submovements. Neuroimage 47:17571766.

Turner RS, Grafton ST, Votaw JR, Delong MR, Hoffman JM (1998) Motor subcircuits mediating the control of movement velocity: a PET study. J Neurophysiol 80:2162-2176.

Turner RS, Desmurget M, Grethe J, Crutcher MD, Grafton ST (2003) Motor subcircuits mediating the control of movement extent and speed. J Neurophysiol 90:3958-3966.

Vaillancourt DE, Mayka MA, Thulborn KR, Corcos DM (2004) Subthalamic nucleus and internal globus pallidus scale with the rate of change of force production in humans. Neuroimage 23:175-186. 